OPEN ACCESS

Edited by:

Ye Zeng,

Sichuan University, China

Reviewed by:

Leonardo Jose de Moura

Carvalho,

Oswaldo Cruz Foundation (Fiocruz),

Brazil

Casper Hempel,

Technical University of Denmark,

Denmark

${ }^{*}$ Correspondence:

J. Brice Weinberg

brice@duke.edu

Specialty section:

This article was submitted to

Signaling,

a section of the journal

Frontiers in Cell and Developmental

Biology

Received: 31 July 2021 Accepted: 27 September 2021

Published: 10 November 2021

Citation:

Bush MA, Anstey NM, Yeo TW,

Florence $S M$, Granger $D L$, Mwaikambo ED and Weinberg JB

(2021) Vascular Dysfunction in Malaria: Understanding the Role

of the Endothelial Glycocalyx.

Front. Cell Dev. Biol. 9:751251.

doi: 10.3389/fcell.2021.751251

\section{Vascular Dysfunction in Malaria: Understanding the Role of the Endothelial Glycocalyx}

\author{
Margaret A. Bush ${ }^{1}$, Nicholas M. Anstey' ${ }^{2}$ Tsin W. Yeo ${ }^{2,3,4}$, Salvatore M. Florence ${ }^{5}$, \\ Donald L. Granger6, Esther D. Mwaikambo ${ }^{5}$ and J. Brice Weinberg ${ }^{7 *}$
}

${ }^{1}$ Duke University School of Nursing and Durham VA Medical Centers, Durham, NC, United States, ${ }^{2}$ Menzies School of Health Research, Charles Darwin University, Darwin, NT, Australia, ${ }^{3}$ Lee Kong Chian School of Medicine, Nanyang Technological University, Singapore, Singapore, ${ }^{4}$ National Centre for Infectious Diseases, Tan Tock Seng Hospital, Singapore, Singapore, ${ }^{5}$ Hubert Kairuki Memorial University, Dar es Salaam, Tanzania, ${ }^{6}$ School of Medicine, University of Utah and Salt Lake City VA Medical Centers, Salt Lake City, UT, United States, ' Duke University School of Medicine and Durham VA Medical Centers, Durham, NC, United States

Malaria caused by Plasmodium falciparum results in over 400,000 deaths annually, predominantly affecting African children. In addition, non-falciparum species including vivax and knowlesi cause significant morbidity and mortality. Vascular dysfunction is a key feature in malaria pathogenesis leading to impaired blood perfusion, vascular obstruction, and tissue hypoxia. Contributing factors include adhesion of infected RBC to endothelium, endothelial activation, and reduced nitric oxide formation. Endothelial glycocalyx (eGC) protects the vasculature by maintaining vessel integrity and regulating cellular adhesion and nitric oxide signaling pathways. Breakdown of eGC is known to occur in infectious diseases such as bacterial sepsis and dengue and is associated with adverse outcomes. Emerging studies using biochemical markers and in vivo imaging suggest that eGC breakdown occurs during Plasmodium infection and is associated with markers of malaria disease severity, endothelial activation, and vascular function. In this review, we describe characteristics of eGC breakdown in malaria and discuss how these relate to vascular dysfunction and adverse outcomes. Further understanding of this process may lead to adjunctive therapy to preserve or restore damaged eGC and reduce microvascular dysfunction and the morbidity/mortality of malaria.

Keywords: glycocalyx, malaria, glycosaminoglycans, endothelium, vascular dysfunction

\section{INTRODUCTION}

In 2019 there were 229 million cases of malaria and 409,000 deaths despite effective anti-parasitic drug therapy (WHO, 2020). The highest malaria disease burden is in Africa, $(94 \%$ of cases and deaths), followed by South and South-East Asia (WHO, 2020). Children $<5$ years account for $\sim 2 / 3$ of deaths (WHO, 2020). Plasmodium falciparum is the malaria responsible for the vast majority of severe illness and deaths globally (WHO, 2020). Plasmodium vivax causes significant morbidity

Abbreviations: ADMA, asymmetric dimethylarginine; Ang-2, angiopoietin-2; BDNF, brain-derived neurotrophic factor; CM, cerebral malaria; CS, chondroitin sulfate; eGC, endothelial glycocalyx; eNOS, endothelial nitric oxide synthase; GAG, glycosaminoglycan; HS, heparan sulfate; HA, hyaluronic acid; iRBC, infected red blood cell; NO, nitric oxide; OPG, osteoprotegerin; PfEMP1, P. falciparum erythrocyte membrane protein 1; RBC, red blood cell; S1P, sphingosine-1-phosphate. 
outside of sub-Saharan Africa, and is recognized as causing delayed severe disease and death (Price et al., 2020). Although less common, the simian parasite Plasmodium knowlesi causes zoonotic malaria in Southeast Asia, which is frequently severe and sometimes fatal (Grigg et al., 2018).

The malaria life cycle in humans starts with the bite of an infected female Anopheles mosquito. Injected sporozoites quickly pass into the liver, multiply asexually within hepatocytes, and develop into merozoites over $\sim 7$ days. After this asymptomatic period, merozoites rupture from the hepatocytes and invade red blood cells (RBC) where they multiply. Rupture of infected $\mathrm{RBC}$ (iRBC) causes fever and release of new merozoites which invade other RBC. This erythrocytic/asexual cycle then repeats (Miller et al., 2013).

Interaction between the $\mathrm{iRBC}$ and the vascular endothelium results in key features of pathogenesis (Miller et al., 2013). In $P$. falciparum infections, iRBC express binding proteins on the RBC membrane (e.g., PfEMP1) and develop knobs that facilitate adherence to vascular endothelium resulting in parasite sequestration in microvessels (Miller et al., 2013). Vascular dysfunction is associated with endothelial activation, impaired perfusion/oxygenation, as well as organ dysfunction. Endothelial activation, microvascular dysfunction, and reduced availability of the endogenous vasodilator nitric oxide (NO) are related to disease severity and outcome (Anstey et al., 1996; Yeo et al., 2007, 2014, 2017). Microvascular sequestration and endothelial activation are independent predictors of impaired perfusion, severe disease, and death (Hanson et al., 2015), but the precise mechanisms of vascular dysfunction in malaria remain unclear.

The endothelial glycocalyx (eGC) serves protective and homeostatic roles in the vasculature (Villalba et al., 2021). Major structural components of the eGC are glycosaminoglycans (GAG), including chondroitin sulfate (CS), heparan sulfate (HS), and hyaluronic acid (HA), and proteoglycans such as syndecans and glypicans (Weinbaum et al., 2021). Of particular interest in malaria, the eGC can modulate cellular adhesion of platelets and iRBC to endothelial cells (Chappell et al., 2014; Hempel et al., 2017; Introini et al., 2018). In placental malaria, CS and syndecan1 support cytoadhesion/sequestration after syncytiotrophoblast denudation (Hromatka et al., 2013; Ayres Pereira et al., 2016; Fried and Duffy, 2017). eGC regulates blood flow-mediated NO production by endothelial NO synthase (eNOS) (Florian et al., 2003; Yen et al., 2015; Weinbaum et al., 2021). Shear stress generated by flowing blood can activate eNOS through a mechano-transduction pathway mediated by eGG (specifically HS and the proteoglycan glypican-1). Downstream effects of this pathway include phosphorylation of PECAM-1 and eNOS, which ultimately results in synthesis of NO (Weinbaum et al., 2021).

Breakdown of eGC occurs in certain infectious diseases in which blood borne pathogens (and host factors they induce) come into contact with eGC. Loss of eGC reported in these diseases relates to pathology, disease severity, and adverse outcomes. In sepsis, blood and urine levels of eGC breakdown products are associated with adverse outcomes including organ dysfunction and mortality (Schmidt et al., 2016; Uchimido et al., 2019). In dengue infection, eGC breakdown is related to increased vascular permeability and disease severity
(Tang et al., 2017). More recently, injury to eGC has been reported in patients with severe COVID-19 infection (Stahl et al., 2020; Queisser et al., 2021).

Given the close overlap of eGC functions with key pathophysiological characteristics of malaria, including cytoadhesion and reduced NO bioavailability, it is logical to suspect potential involvement of the eGC in malaria (Figure 1). The purpose of this review is to summarize evidence for eGC breakdown in malaria and describe how this may relate to pathogenesis of microvascular dysfunction and adverse clinical outcomes. Pre-clinical and clinical studies in malaria are listed in Table 1.

\section{EXPERIMENTAL ANIMAL STUDIES OF THE ENDOTHELIAL GLYCOCALYX IN MALARIA}

Hempel et al. (2014) first reported loss of eGC in malaria using a mouse model of cerebral malaria (CM). Plasma GAG levels were significantly increased, and transmission electron microscopy showed complete or partial loss of eGC in mice with CM (Hempel et al., 2014). Daily plasma GAG levels suggested that shedding of eGC begins early in the infection (Hempel et al., 2014). Follow-up studies with the murine experimental $\mathrm{CM}$ model demonstrated loss of GAG (HA, HS, CS) and proteoglycans (syndecan-1 and 4) in the brain with corresponding increases in plasma concentrations of these eGC constituents, as well as an increase in vascular permeability (Hempel et al., 2019). The authors showed that dexamethasone or antithrombin-3 prevent the eGC loss and certain disease manifestations (Hempel et al., 2019). While the murine cerebral malaria model is commonly used in malaria research, questions have been raised about suitability of the model for translation of results to clinical disease in humans (White et al., 2010; Craig et al., 2012). Challenges with the model include use of different species of parasite and innate differences between mice and humans in disease manifestations and pathophysiology (notably characteristics of adherence of iRBC to endothelial cells and degree of sequestration) (White et al., 2010; Craig et al., 2012). While certain treatments have shown to be effective in the mouse CM model, thus far the findings in mice have not proven helpful in identifying beneficial therapeutics for adjunctive treatment in humans.

\section{CYTOADHESION STUDIES}

Several in vitro studies have demonstrated that cytoadhesion is increased upon loss of eGC. Hempel et al. (2017) studied in vitro binding to Chinese hamster ovary cells expressing CD36, a known binding receptor for falciparum-infected RBCs. They found that cytoadhesion was inhibited by presence of eGC, suggesting that loss of eGC may have a role in cellular adhesion process by facilitating the ability to interact with binding receptors such as CD36.

Using a "microfluidic organ-on-chip" model designed to mimic human microvessels, adhesion of iRBC to human 

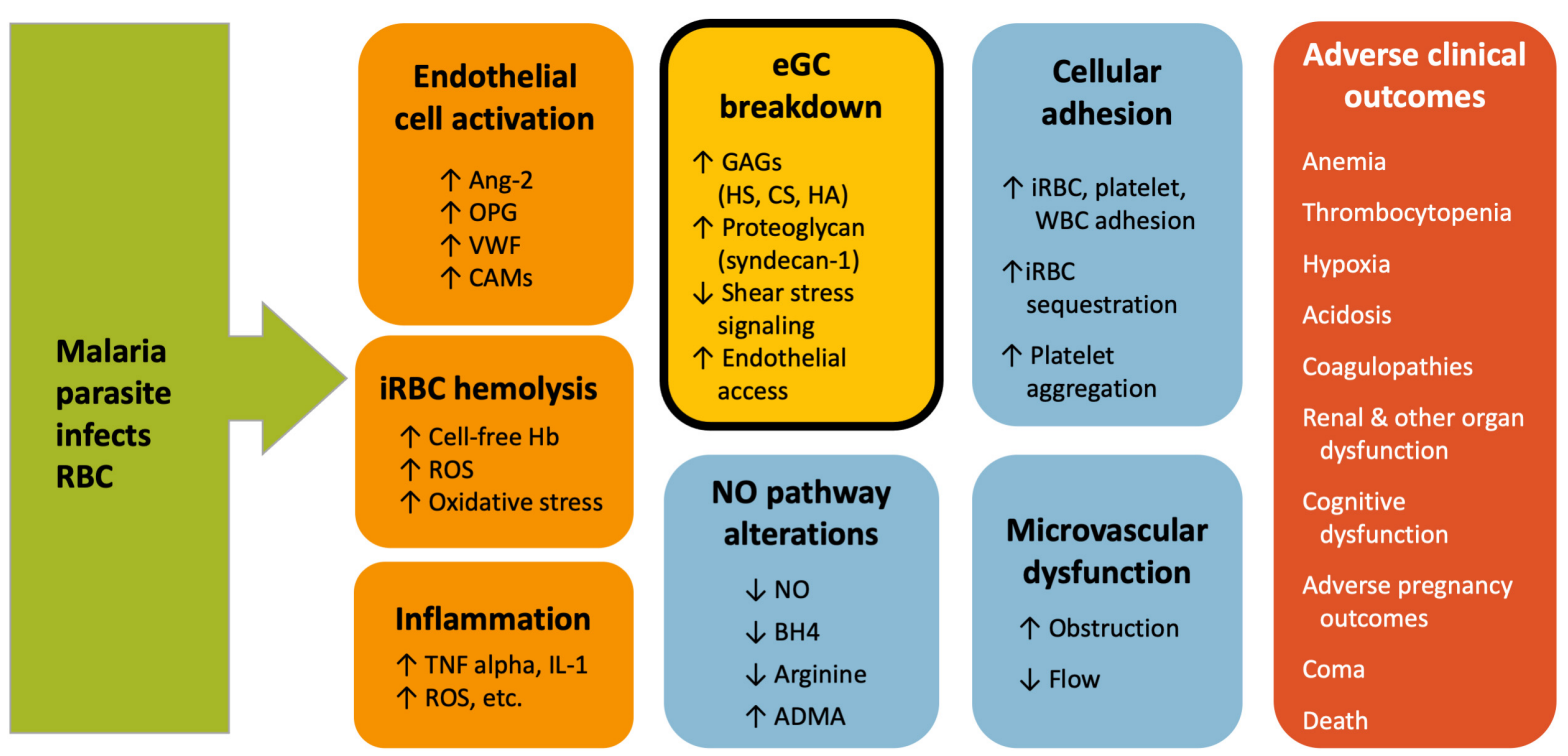

FIGURE 1 | Vascular dysfunction in Malaria. Multiple vascular events contribute to malaria pathogenesis and lead to adverse clinical outcomes. The exact mechanism and sequence of events is not known, but early events include endothelial cell activation and rupture of infected RBC. These lead to endothelial glycocalyx breakdown, reduced NO, cellular adhesion and microvascular dysfunction. Endothelial glycocalyx breakdown (shown in the gold, bolded box) is associated with multiple markers of malaria disease severity and may play a central role in different aspects of malaria pathogenesis. ADMA, asymmetric dimethylarginine; Ang-2, angiopoietin-2; BH4, tetrahydrobiopterin; CAMs, cell adhesion molecules; CS, chondroitin sulfate; eGC, endothelial glycocalyx; GAG, glycosaminoglycan; Hb, hemoglobin; HA, hyaluronic acid; HS, heparan sulfate; iRBC, infected red blood cell; NO, nitric oxide; OPG, osteoprotegerin; ROS, reactive oxygen species; S1P, sphingosine-1-phosphate; WWF, von Willebrand factor.

umbilical vein endothelial cells (HUVEC) was enhanced following enzymatic degradation of the eGC, compared to adhesion of healthy RBC (Introini et al., 2018). Altered dynamics of the iRBC, including flipping and decreased flow velocity, were also reported, consistent with altered hemodynamics that contribute to cytoadhesion of $\mathrm{iRBC}$ to endothelium (Introini et al., 2018). These cytoadhesion studies suggest that the eGC is integral in regulating cytoadhesion of iRBC.

\section{CLINICAL STUDIES IN HUMAN MALARIA}

The eGC is required for induction of NO formation by blood flow over endothelial cells (Florian et al., 2003; Yen et al., 2015; Weinbaum et al., 2021). Our prior studies have documented that host NO production is associated with protection against severe disease in falciparum malaria (Anstey et al., 1996; Yeo et al., 2007, 2014), vivax malaria (Barber et al., 2016), and knowlesi malaria (Barber et al., 2018). Thus, we performed studies to determine if eGC is damaged in humans with malaria from each of Plasmodium species known to cause severe disease.

We initially reported evidence of degradation of the eGC in falciparum malaria in 129 Indonesian adults (Yeo et al., 2019b) and 100 Tanzanian children (Yeo et al., 2019a); and subsequently in 146 Tanzanian children (Bush et al., 2021). In both children and adults, there were significant increases in total sulfated GAG in urine. We further characterized the elevated urinary GAG constituents by mass spectrometry and found predominantly HS and CS (but not dermatan sulfate) to be elevated in malaria patients (Yeo et al., 2019a,b), consistent with the composition of GC from the endothelium (Weinbaum et al., 2007). In children, longitudinal analyses showed that urine total GAG levels decreased daily with treatment and normalized by Day 3 (Yeo et al., 2019a). Total GAG were significantly associated with markers of malaria disease severity including parasitemia, parasite biomass, cell-free hemoglobin, and plasma TNF (Yeo et al., 2019a,b). Markers of endothelial activation such as ICAM-1, E-selectin, and Ang-2 were also associated with eGC breakdown products (Yeo et al., 2019b). Breakdown of eGC may expose ICAM-1 and E-selectin, endothelial adhesion molecules implicated in cytoadherence of $P$. falciparum, and exacerbate microvascular sequestration (Hempel et al., 2017; Introini et al., 2018). In both adults and children, measures of NO bioavailability and vascular function were inversely related to the eGC breakdown products (Yeo et al., 2019a,b). In adults, degradation of eGC was associated with mortality (Yeo et al., 2019b). The plasma core protein syndecan-1 was also significantly elevated in adult patients and associated with various markers of disease severity (Yeo et al., 2019b). Degradation of eGC has been implicated in the pathogenesis of acute kidney injury in sepsis (Schmidt et al., 2016). The greater magnitude of eGC degradation in acute kidney injury seen in severe falciparum malaria (Yeo et al., 2019b) supports a role for glomerular eGC degradation in the pathogenesis of acute kidney injury in malaria.

Lyimo et al. (2020) used incident dark field imaging of the microvasculature in the buccal region of the mouth along with biochemical measures including GAG and syndecan-1 to demonstrate eGC loss in African children with malaria. Imaging 
TABLE 1 | Studies of eGC in malaria.

\begin{tabular}{ll}
\hline Pre-clinical & Clinical \\
\hline $\begin{array}{l}\text { Mouse cerebral malaria (Hempel } \\
\text { et al., 2014) }\end{array}$ & P. falciparum in Indonesian adults \\
Mouse cerebral malaria (Hempel & Peo et al., 2019b) \\
et al., 2019) & (Yeo et al., 2019a) \\
CD36 binding in vitro (Hempel & P. falciparum in Tanzanian children \\
et al., 2017) & (Lyimo et al., 2020) \\
iRBC cytoadhesion and flow & P. falciparum or P. vivax, \\
characteristics (Introini et al., 2018) & experimentally induced in human \\
& volunteers (Woodford et al., 2020) \\
& P. vivax and $P$. knowlesi in \\
& Malaysian adults (Barber et al., \\
& 2021) \\
& P. falciparum in Tanzanian children \\
& (Bush et al., 2021)
\end{tabular}

was used to calculate the perfused boundary region (PBR), a measure used to assess the ability of perfused RBC to penetrate the eGC. The PBR measurement for children with either uncomplicated or severe malaria was significantly increased compared with healthy children, supporting breakdown of the eGC barrier (Lyimo et al., 2020). Additional findings detected on imaging in the malaria patients included impaired vascular integrity, stagnant erythrocytes external to blood vessels, perivascular hemorrhages, and sequestration of late-stage parasites within vessels (Lyimo et al., 2020). Similar to other clinical studies, biochemical markers of eGC loss were elevated in the malaria patients. However in these studies, measures of eGC degradation were slower to recover, with eGC integrity remaining impaired for at least 2 weeks. Biomarkers of endothelial activation in plasma (i.e., angiopoietin-1/2 and thrombomodulin) were also significantly altered in malaria patients (Lyimo et al., 2020).

While most studies to date have focused on breakdown of eGC in falciparum malaria, there is also evidence for breakdown of eGC in vivax and knowlesi malaria (Barber et al., 2021). In Malaysian adults with either vivax or knowlesi infection, levels of eGC breakdown products were significantly increased compared to healthy controls and in knowlesi malaria, were significantly correlated with disease severity (Barber et al., 2021). In knowlesi malaria, there is also significant correlation with biomarkers of endothelial activation [angiopoietin-2 and osteoprotegerin (OPG)] and measures of microvascular function. In the patients with knowlesi malaria, the eGC breakdown marker syndecan-1 was also significantly related to acute kidney injury, supporting the notion that glomerular glycocalyx degradation is a key mechanism of acute kidney injury in malaria and other acute infections.

\section{CLINICAL EVIDENCE IN EXPERIMENTALLY INDUCED HUMAN MALARIA}

The study of healthy volunteers inoculated with malaria parasites presents a unique opportunity to observe events early in infection, compared with clinical trials in which patients have had infection for some time prior to diagnosis. Subjects are inoculated with infected $\mathrm{RBC}$ following which parasitemia develops. At onset of clinical signs or $\sim 7$ days after inoculation, the patients receive antimalarial drug treatment. In 45 healthy subjects inoculated with $P$. falciparum or $P$. vivax, biomarkers of endothelial activation, including von Willebrand factor and OPG, increased from baseline to the start of antimalarial treatment, after which levels declined back to baseline (Woodford et al., 2020). In the P. falciparum group, Ang-2 increased significantly. However at these earlier timepoints of infection, there were no changes observed in biochemical markers of eGC breakdown, abnormalities using sidestream dark field microscopy of sublingual vessels, or microvascular function as assessed by peripheral arterial tonometry or near-infrared spectroscopy (Woodford et al., 2020). Our study suggests that endothelial activation precedes eGC breakdown and vascular dysfunction, and it also supports potential involvement of Ang-2 as a mediator of eGC breakdown.

\section{MECHANISMS OF ENDOTHELIAL GLYCOCALYX DEGRADATION IN MALARIA}

While the mechanisms of eGC loss in malaria are not certain, a number of mediators are implicated. There is evidence that angiopoietin-2 contributes to eGC degradation. Elevated plasma levels of Ang-2 have been demonstrated in malaria and are associated with endothelial activation and disease severity (Yeo et al., 2008; Conroy et al., 2009). In falciparum malaria (Yeo et al., 2019b), vivax malaria (Barber et al., 2021), knowlesi malaria (Barber et al., 2021), and other disease settings including sepsis (Beurskens et al., 2020), Ang-2 has been associated with eGC loss, independent of other potential mediators. And in cultured endothelial cells, Ang-2 induces a heparanasedependent degradation of eGC (Lukasz et al., 2017). Loss of eGC results when the breakdown predominates over new eGC synthesis. Heparanase as well as other substances (e.g., membrane or matrix metalloproteases, thrombin, plasmin, and hyaluronidases) have been implicated as potential "sheddases" that are responsible for eGC breakdown clinically (Becker et al., 2015; Iba and Levy, 2019). Endothelial NOS (eNOS) can prevent the induction of heparanases, with heparanase induced by the eNOS inhibitor asymetric dimethylarginine (ADMA) (Garsen et al., 2016). The eNOS-inhibitors OPG and ADMA are elevated in both falciparum and knowlesi malaria (Yeo et al., 2010; Barber et al., 2021), and both are associated with eGC degradation in malaria (Barber et al., 2021). ADMA and OPG may therefore contribute to induction of heparanase in malaria and to eGC degradation. Oxidative stress is elevated in severe malaria (Rubach et al., 2015; Yeo et al., 2015), and can also mediate glycocalyx breakdown (Pillinger and Kam, 2017). Hemolysis in malaria results in circulating, cell-free plasma hemoglobin that is associated with oxidative stress (Plewes et al., 2017) and eGC breakdown, independent of disease severity (Yeo et al., 2019b). Heme-mediated oxidative stress 
likely also contributes to glycocalyx breakdown in malaria. The lipid mediator, sphingosine-1-phosphate (S1P), protects against eGC damage by inhibiting syndecan-1 shedding and increasing glycocalyx synthesis (Zeng et al., 2014). S1P is decreased in malaria (Finney et al., 2011; Punsawad and Viriyavejakul, 2017; Yeo et al., 2019b; Barber et al., 2021), and in falciparum malaria is inversely associated with eGC degradation (Yeo et al., 2019b). This suggests that reduced S1P may also contribute toward loss of glycocalyx integrity in malaria. Since erythrocytes are a major source of S1P, malaria-associated anemia could enhance deficiencies in S1P signaling pathways.

Further understanding of which sheddases are involved in malaria could identify potential pharmacologic targets leading to new adjunctive treatments. Better understanding of the mechanisms will also help determine how eGC loss affects factors related to both eGC function and malaria pathogenesis (including $\mathrm{NO}$ formation in response to shear stress of flowing blood and the processes of cellular adhesion to endothelium by leukocytes, platelets and iRBC).

An interesting question is whether the eGC breakdown products could themselves have detrimental effects and contribute to pathogenesis, rather than being simply being inert, circulating GAGs and proteoglycans. This concept has been reported in sepsis in which GAG are linked to cognitive sequelae. In a mouse model, circulating GAG fragments were shown to cross the blood brain barrier, bind to brain-derived neurotrophic factor (BDNF), and inhibit BDNF-mediated longterm potentiation in the hippocampus, a mechanism underlying memory (Hippensteel et al., 2019). Particular sulfation patterns of HS were associated with better binding to BDNF (Hippensteel et al., 2019). Interestingly, in patients with sepsis in the intensive care unit, these same sulfation patterns in plasma HS predicted cognitive impairment lasting up to 2 weeks after discharge (Hippensteel et al., 2019). Lyimo et al. showed that in children with cerebral malaria, plasma concentrations of HS were significantly higher in those with deeper coma (Lyimo et al., 2020). It is possible that eGC degradation not only exacerbates parasite sequestration and microvascular dysfunction in cerebral malaria, but through elevated circulating HS, may compound coma severity in cerebral malaria. Another example of pathological changes related to breakdown products themselves is in COVID-19 in which endothelial barrier dysfunction can be induced by HA fragments (Queisser et al., 2021).

A therapeutic that could prevent loss or promote formation of eGC could potentially be used as adjunctive therapy in patients with malaria. There are a number of proposed therapeutics to ameliorate eGC loss by mechanisms such as preventing shedding, inhibiting putative sheddases, or regenerating eGC

\section{REFERENCES}

Anstey, N. M., Weinberg, J. B., Hassanali, M. Y., Mwaikambo, E. D., Manyenga, D., Misukonis, M. A., et al. (1996). Nitric oxide in Tanzanian children with malaria: inverse relationship between malaria severity and nitric oxide production/nitric oxide synthase type 2 expression. J. Exp. Med. 184, 557-567.

Ayres Pereira, M., Mandel Clausen, T., Pehrson, C., Mao, Y., Resende, M., Daugaard, M., et al. (2016). Placental sequestration of plasmodium falciparum (e.g., albumin, sulodexide, sevoflurane, rhamnan sulfate) (Iba and Levy, 2019; Weinbaum et al., 2021). Data regarding these agents are mainly based on experimental animal studies, and there is no clinical evidence to support their clinical use currently. Of relevance to malaria, sevuparin is a modified low molecular weight heparin-like molecule without anticoagulant properties and with close similarity to the glycosaminoglycan HS. Sevuparin and other heparin analogs inhibit rosetting (binding of iRBCs and RBCs) and cytoadhesion of iRBC to endothelium in vitro (Vogt et al., 2006; Leitgeb et al., 2011; Saiwaew et al., 2017). In a Phase I/II clinical trial in patients with uncomplicated malaria, sevuparin inhibited parasite invasion of $\mathrm{RBC}$ and reversed sequestration of $\mathrm{iRBC}$ (Leitgeb et al., 2017).

\section{CONCLUSION}

There is growing evidence that loss of eGC is associated with vascular dysfunction and disease severity in malaria. This loss may lead to multiple vascular events and adverse clinical outcomes in malaria (Hempel et al., 2016; Georgiadou and Cunnington, 2019). Many questions remain including the need for further understanding of the mechanisms underlying eGC breakdown in malaria. We need to identify pathways responsible for eGC breakdown, e.g., the initial steps and role of the parasite or host factors as triggers; whether the breakdown products themselves are detrimental contributors to pathology; and the relation and kinetics of eGC synthesis vs. breakdown in malaria. Clinically, it will be important to determine if rapid assays of eGC breakdown products (e.g., urine total GAG) can provide helpful prognostic and treatment insights, and whether adjunctive agents that prevent loss or replace eGC can ameliorate or prevent severe disease.

\section{AUTHOR CONTRIBUTIONS}

All authors listed have made a substantial, direct and intellectual contribution to the work, and approved it for publication.

\section{FUNDING}

This work was supported by the US National Institutes of Health, National Heart, Lung, and Blood Institute Grant R01 HL13076301 and the VA Research Service (JW). NA was supported by the National Health and Medical Research Council (1135820).

malaria parasites is mediated by the interaction between VAR2CSA and chondroitin sulfate a on syndecan-1. PLoS Pathog. 12:e1005831. doi: 10.1371/ journal.ppat.1005831

Barber, B. E., Grigg, M. J., Piera, K. A., Chen, Y., William, T., Weinberg, J. B., et al. (2021). Endothelial glycocalyx degradation and disease severity in Plasmodium vivax and Plasmodium knowlesi malaria. Sci. Rep. 11:9741.

Barber, B. E., Grigg, M. J., Piera, K. A., William, T., Cooper, D. J., Plewes, K., et al. (2018). Intravascular haemolysis in severe Plasmodium knowlesi malaria: 
association with endothelial activation, microvascular dysfunction, and acute kidney injury. Emerg. Microbes Infect. 7:106. doi: 10.1038/s41426-018-0105-2

Barber, B. E., William, T., Grigg, M. J., Piera, K. A., Chen, Y., Wang, H., et al. (2016). Nitric oxide-dependent endothelial dysfunction and reduced arginine bioavailability in Plasmodium vivax malaria but no greater increase in intravascular hemolysis in severe disease. J. Infect. Dis. 214, 1557-1564. doi: 10.1093/infdis/jiw427

Becker, B. F., Jacob, M., Leipert, S., Salmon, A. H., and Chappell, D. (2015). Degradation of the endothelial glycocalyx in clinical settings: searching for the sheddases. Br. J. Clin. Pharmacol. 80, 389-402. doi: 10.1111/bcp.12629

Beurskens, D. M., Bol, M. E., Delhaas, T., van de Poll, M. C., Reutelingsperger, C. P., Nicolaes, G. A., et al. (2020). Decreased endothelial glycocalyx thickness is an early predictor of mortality in sepsis. Anaesth. Intensive Care 48, 221-228. doi: 10.1177/0310057X20916471

Bush, M. A., Florence, S. M., Yeo, T. W., Kalingonji, A. R., Chen, Y., Granger, D. L., et al. (2021). Degradation of endothelial glycocalyx in Tanzanian children with falciparum malaria. FASEB J. 35:e21805. doi: 10.1096/fj.202100277RR

Chappell, D., Brettner, F., Doerfler, N., Jacob, M., Rehm, M., Bruegger, D., et al. (2014). Protection of glycocalyx decreases platelet adhesion after ischaemia/reperfusion: an animal study. Eur. J. Anaesthesiol. 31, 474-481. doi: 10.1097/EJA.0000000000000085

Conroy, A. L., Lafferty, E. I., Lovegrove, F. E., Krudsood, S., Tangpukdee, N., Liles, W. C., et al. (2009). Whole blood angiopoietin-1 and -2 levels discriminate cerebral and severe (non-cerebral) malaria from uncomplicated malaria. Malar. J. 8:295. doi: 10.1186/1475-2875-8-295

Craig, A. G., Grau, G. E., Janse, C., Kazura, J. W., Milner, D., Barnwell, J. W., et al. (2012). The role of animal models for research on severe malaria. PLoS Pathog. 8:e1002401. doi: 10.1371/journal.ppat.1002401

Finney, C. A., Hawkes, C. A., Kain, D. C., Dhabangi, A., Musoke, C., CsertiGazdewich, C., et al. (2011). S1P is associated with protection in human and experimental cerebral malaria. Mol. Med. 17, 717-725. doi: 10.2119/molmed. 2010.00214

Florian, J. A., Kosky, J. R., Ainslie, K., Pang, Z., Dull, R. O., and Tarbell, J. M. (2003). Heparan sulfate proteoglycan is a mechanosensor on endothelial cells. Circ. Res. 93, e136-e142. doi: 10.1161/01.RES.0000101744.47866.D5

Fried, M., and Duffy, P. E. (2017). Malaria during pregnancy. Cold Spring Harb. Perspect. Med. 7:a025551. doi: 10.1101/cshperspect.a025551

Garsen, M., Rops, A. L., Li, J., van Beneden, K., van den Branden, C., Berden, J. H., et al. (2016). Endothelial nitric oxide synthase prevents heparanase induction and the development of proteinuria. PLoS One 11:e0160894. doi: 10.1371/ journal.pone.0160894

Georgiadou, A., and Cunnington, A. J. (2019). Shedding of the vascular endothelial glycocalyx: a common pathway to severe malaria? Clin. Infect. Dis. 69, 17211723. doi: $10.1093 / \mathrm{cid} / \mathrm{ciz} 043$

Grigg, M. J., William, T., Barber, B. E., Rajahram, G. S., Menon, J., Schimann, E., et al. (2018). Age-related clinical spectrum of plasmodium knowlesi malaria and predictors of severity. Clin. Infect. Dis. 67, 350-359. doi: 10.1093/cid/ciy065

Hanson, J., Lee, S. J., Hossain, M. A., Anstey, N. M., Charunwatthana, P., Maude, R. J., et al. (2015). Microvascular obstruction and endothelial activation are independently associated with the clinical manifestations of severe falciparum malaria in adults: an observational study. BMC Med. 13:122. doi: 10.1186/ s12916-015-0365-9

Hempel, C., Hyttel, P., and Kurtzhals, J. A. (2014). Endothelial glycocalyx on brain endothelial cells is lost in experimental cerebral malaria. J. Cereb. Blood Flow Metab. 34, 1107-1110. doi: 10.1038/jcbfm.2014.79

Hempel, C., Pasini, E. M., and Kurtzhals, J. A. (2016). Endothelial glycocalyx: shedding light on malaria pathogenesis. Trends Mol. Med. 22, 453-457. doi: 10.1016/j.molmed.2016.04.004

Hempel, C., Sporring, J., and Kurtzhals, J. A. L. (2019). Experimental cerebral malaria is associated with profound loss of both glycan and protein components of the endothelial glycocalyx. FASEB J. 33, 2058-2071. doi: 10.1096/fj. 201800657R

Hempel, C., Wang, C. W., Kurtzhals, J. A. L., and Staalso, T. (2017). Binding of plasmodium falciparum to CD36 can be shielded by the glycocalyx. Malar. J. 16:193. doi: 10.1186/s12936-017-1844-6

Hippensteel, J. A., Anderson, B. J., Orfila, J. E., McMurtry, S. A., Dietz, R. M., Su, G., et al. (2019). Circulating heparan sulfate fragments mediate septic cognitive dysfunction. J. Clin. Invest. 129, 1779-1784. doi: 10.1172/JCI124485
Hromatka, B. S., Ngeleza, S., Adibi, J. J., Niles, R. K., Tshefu, A. K., and Fisher, S. J. (2013). Histopathologies, immunolocalization, and a glycan binding screen provide insights into Plasmodium falciparum interactions with the human placenta. Biol. Reprod. 88:154. doi: 10.1095/biolreprod.112.106195

Iba, T., and Levy, J. H. (2019). Derangement of the endothelial glycocalyx in sepsis. J. Thromb. Haemost. 17, 283-294. doi: 10.1111/jth.14371

Introini, V., Carciati, A., Tomaiuolo, G., Cicuta, P., and Guido, S. (2018). Endothelial glycocalyx regulates cytoadherence in Plasmodium falciparum malaria. J. R. Soc. Interface 15:20180773. doi: 10.1098/rsif.2018.0773

Leitgeb, A. M., Blomqvist, K., Cho-Ngwa, F., Samje, M., Nde, P., Titanji, V., et al. (2011). Low anticoagulant heparin disrupts Plasmodium falciparum rosettes in fresh clinical isolates. Am. J. Trop. Med. Hyg. 84, 390-396. doi: 10.4269/ajtmh. 2011.10-0256

Leitgeb, A. M., Charunwatthana, P., Rueangveerayut, R., Uthaisin, C., Silamut, K., Chotivanich, K., et al. (2017). Inhibition of merozoite invasion and transient de-sequestration by sevuparin in humans with Plasmodium falciparum malaria. PLoS One 12:e0188754. doi: 10.1371/journal.pone.0188754

Lukasz, A., Hillgruber, C., Oberleithner, H., Kusche-Vihrog, K., Pavenstadt, H., Rovas, A., et al. (2017). Endothelial glycocalyx breakdown is mediated by angiopoietin-2. Cardiovasc. Res. 113, 671-680. doi: 10.1093/cvr/c vx023

Lyimo, E., Haslund, L. E., Ramsing, T., Wang, C. W., Efunshile, A. M., Manjurano, A., et al. (2020). In vivo imaging of the buccal mucosa shows loss of the endothelial glycocalyx and perivascular hemorrhages in pediatric Plasmodium falciparum Malaria. Infect. Immun. 88, e679-e619. doi: 10.1128/IAI.00679-19

Miller, L. H., Ackerman, H. C., Su, X. Z., and Wellems, T. E. (2013). Malaria biology and disease pathogenesis: insights for new treatments. Nat. Med. 19, 156-167. doi: 10.1038/nm.3073

Pillinger, N. L., and Kam, P. (2017). Endothelial glycocalyx: basic science and clinical implications. Anaesth. Intensive Care 45, 295-307. doi: 10.1177/ $0310057 \mathrm{X} 1704500305$

Plewes, K., Kingston, H. W. F., Ghose, A., Maude, R. J., Herdman, M. T., Leopold, S. J., et al. (2017). Cell-free hemoglobin mediated oxidative stress is associated with acute kidney injury and renal replacement therapy in severe falciparum malaria: an observational study. BMC Infect. Dis. 17:313. doi: 10.1186/s12879017-2373-1

Price, R. N., Commons, R. J., Battle, K. E., Thriemer, K., and Mendis, K. (2020). Plasmodium vivax in the Era of the Shrinking P. falciparum Map. Trends Parasitol. 36, 560-570. doi: 10.1016/j.pt.2020.03.009

Punsawad, C., and Viriyavejakul, P. (2017). Reduction in serum sphingosine 1phosphate concentration in malaria. PLoS One 12:e0180631. doi: 10.1371/ journal.pone.0180631

Queisser, K. A., Mellema, R. A., Middleton, E. A., Portier, I., Manne, B. K., Denorme, F., et al. (2021). COVID-19 generates hyaluronan fragments that directly induce endothelial barrier dysfunction. JCI Insight 6:147472. doi: 10. 1172/jci.insight. 147472

Rubach, M. P., Mukemba, J., Florence, S., Lopansri, B. K., Hyland, K., Volkheimer, A. D., et al. (2015). Impaired systemic tetrahydrobiopterin bioavailability and increased oxidized biopterins in pediatric falciparum malaria: association with disease severity. PLoS Pathog. 11:e1004655. doi: 10.1371/journal.ppat.1004655

Saiwaew, S., Sritabal, J., Piaraksa, N., Keayarsa, S., Ruengweerayut, R., Utaisin, C., et al. (2017). Effects of sevuparin on rosette formation and cytoadherence of Plasmodium falciparum infected erythrocytes. PLoS One 12:e0172718. doi: 10.1371/journal.pone.0172718

Schmidt, E. P., Overdier, K. H., Sun, X., Lin, L., Liu, X., Yang, Y., et al. (2016). Urinary glycosaminoglycans predict outcomes in septic shock and acute respiratory distress syndrome. Am. J. Respir. Crit. Care Med. 194, 439-449. doi: 10.1164/rccm.201511-2281OC

Stahl, K., Gronski, P. A., Kiyan, Y., Seeliger, B., Bertram, A., Pape, T., et al. (2020). Injury to the endothelial glycocalyx in critically ill patients with COVID-19. Am. J. Respir. Crit. Care Med. 202, 1178-1181. doi: 10.1164/rccm.202007-2676LE

Tang, T. H., Alonso, S., Ng, L. F., Thein, T. L., Pang, V. J., Leo, Y. S., et al. (2017). Increased serum hyaluronic acid and heparan sulfate in dengue fever: association with plasma leakage and disease severity. Sci. Rep. 7:46191. doi: $10.1038 /$ srep46191

Uchimido, R., Schmidt, E. P., and Shapiro, N. I. (2019). The glycocalyx: a novel diagnostic and therapeutic target in sepsis. Crit. Care 23:16. doi: 10.1186/ s13054-018-2292-6 
Villalba, N., Baby, S., and Yuan, S. Y. (2021). The endothelial glycocalyx as a double-edged sword in microvascular homeostasis and pathogenesis. Front. Cell Dev. Biol. 9:711003. doi: 10.3389/fcell.2021.711003

Vogt, A. M., Pettersson, F., Moll, K., Jonsson, C., Normark, J., Ribacke, U., et al. (2006). Release of sequestered malaria parasites upon injection of a glycosaminoglycan. PLoS Pathog. 2:e100. doi: 10.1371/journal.ppat.00 20100

Weinbaum, S., Cancel, L. M., Fu, B. M., and Tarbell, J. M. (2021). The glycocalyx and its role in vascular physiology and vascular related diseases. Cardiovasc. Eng. Technol. 12, 37-71. doi: 10.1007/s13239-020-00485-9

Weinbaum, S., Tarbell, J. M., and Damiano, E. R. (2007). The structure and function of the endothelial glycocalyx layer. Annu. Rev. Biomed. Eng. 9, 121167. doi: 10.1146/annurev.bioeng.9.060906.151959

White, N. J., Turner, G. D. I, Medana, M., Dondorp, A. M., and Day, N. P. (2010). The murine cerebral malaria phenomenon. Trends Parasitol. 26, 11-15. doi: $10.1016 /$ j.pt.2009.10.007

WHO (2020). World Malaria Report 2020. Geneva: World Health Organization.

Woodford, J., Yeo, T. W., Piera, K. A., Butler, K., Weinberg, J. B., McCarthy, J. S., et al. (2020). Early endothelial activation precedes glycocalyx degradation and microvascular dysfunction in experimentally induced plasmodium falciparum and plasmodium vivax infection. Infect. Immun. 88, e895-e819. doi: 10.1128/IAI.00 895-19

Yen, W., Cai, B., Yang, J., Zhang, L., Zeng, M., Tarbell, J. M., et al. (2015). Endothelial surface glycocalyx can regulate flow-induced nitric oxide production in microvessels in vivo. PLoS One 10:e0117133. doi: 10.1371/ journal.pone.0117133

Yeo, T. W., Florence, S. M., Kalingonji, A. R., Chen, Y., Granger, D. L., Anstey, N. M., et al. (2017). Decreased microvascular function in tanzanian children with severe and uncomplicated falciparum malaria. Open Forum Infect. Dis. 4:ofx079. doi: 10.1093/ofid/ofx079

Yeo, T. W., Lampah, D. A., Gitawati, R., Tjitra, E., Kenangalem, E., McNeil, Y. R., et al. (2007). Impaired nitric oxide bioavailability and L-arginine reversible endothelial dysfunction in adults with falciparum malaria. J. Exp. Med. 204, 2693-2704. doi: 10.1084/jem.20070819

Yeo, T. W., Lampah, D. A., Gitawati, R., Tjitra, E., Kenangalem, E., Piera, K., et al. (2008). Angiopoietin-2 is associated with decreased endothelial nitric oxide and poor clinical outcome in severe falciparum malaria. Proc. Natl. Acad. Sci. U.S.A 105, 17097-17102. doi: 10.1073/pnas.0805782105

Yeo, T. W., Lampah, D. A., Kenangalem, E., Tjitra, E., Price, R. N., Weinberg, J. B., et al. (2015). Impaired systemic tetrahydrobiopterin bioavailability and increased dihydrobiopterin in adult falciparum malaria: association with disease severity, impaired microvascular function and increased endothelial activation. PLoS Pathog. 11:e1004667. doi: 10.1371/journal.ppat.10 04667

Yeo, T. W., Lampah, D. A., Kenangalem, E., Tjitra, E., Weinberg, J. B., Granger, D. L., et al. (2014). Decreased endothelial nitric oxide bioavailability, impaired microvascular function, and increased tissue oxygen consumption in children with falciparum malaria. J. Infect. Dis. 210, 1627-1632. doi: 10.1093/infdis/ jiu308

Yeo, T. W., Lampah, D. A., Tjitra, E., Gitawati, R., Darcy, C. J., Jones, C., et al. (2010). Increased asymmetric dimethylarginine in severe falciparum malaria: association with impaired nitric oxide bioavailability and fatal outcome. PLoS Pathog. 6:e1000868. doi: 10.1371/journal.ppat.1000868

Yeo, T. W., Weinberg, J. B., Lampah, D. A., Kenangalem, E., Bush, P., Chen, Y., et al. (2019b). Glycocalyx breakdown is associated with severe disease and fatal outcome in Plasmodium falciparum malaria. Clin. Infect. Dis. 69, 1712-1720. doi: $10.1093 / \mathrm{cid} / \mathrm{ciz} 038$

Yeo, T. W., Bush, P. A., Chen, Y., Young, S. P., Zhang, H., Millington, D. S., et al. (2019a). Glycocalyx breakdown is increased in African children with cerebral and uncomplicated falciparum malaria. FASEB J. 33, 14185-14193. doi: 10.1096/fj.201901048RR

Zeng, Y., Adamson, R. H., Curry, F. R., and Tarbell, J. M. (2014). Sphingosine-1phosphate protects endothelial glycocalyx by inhibiting syndecan-1 shedding. Am. J. Physiol. Heart Circ. Physiol. 306, H363-H372. doi: 10.1152/ajpheart. 00687.2013

Conflict of Interest: The authors declare that the research was conducted in the absence of any commercial or financial relationships that could be construed as a potential conflict of interest.

Publisher's Note: All claims expressed in this article are solely those of the authors and do not necessarily represent those of their affiliated organizations, or those of the publisher, the editors and the reviewers. Any product that may be evaluated in this article, or claim that may be made by its manufacturer, is not guaranteed or endorsed by the publisher.

Copyright (c) 2021 Bush, Anstey, Yeo, Florence, Granger, Mwaikambo and Weinberg. This is an open-access article distributed under the terms of the Creative Commons Attribution License (CC BY). The use, distribution or reproduction in other forums is permitted, provided the original author(s) and the copyright owner(s) are credited and that the original publication in this journal is cited, in accordance with accepted academic practice. No use, distribution or reproduction is permitted which does not comply with these terms. 